\title{
P144 Augmentation Index is not a Reliable Measure of Wave Reflections in Patients with Severe Aortic Stenosis
}

\author{
Stamatia Pagoulatou ${ }^{1, *}$, Nikolaos Stergiopulos ${ }^{1}$, Vasiliki Bikia ${ }^{1}$, Georgios Rovas ${ }^{1}$, Hajo Müller², Stéphane Noble ${ }^{2}$, \\ Dionysios Adamopoulos ${ }^{2}$
}

${ }^{1}$ Ecole Polytechnique Fédérale de Lausanne (EPFL), Lausanne, Switzerland

${ }^{2}$ Geneva University Hospitals (HUG), Geneva, Switzerland

\begin{abstract}
Background: Augmentation index (AIx) serves as an independent predictor of cardiovascular events and mortality. It is often clinically used as a measure of arterial stiffness and reflections, a practice that was recently challenged. In this work, we sought to investigate the association between AIx and wave reflections in patients with severe aortic stenosis.

Methods: The final cohort comprised of 18 subjects (86 \pm 4 years, $28 \%$ males) with severe aortic stenosis who underwent a Transcatheter Aortic Valve Implantation (TAVI). Invasive aortic and left ventricular pressure waveforms were obtained simultaneously before and after the TAVI. Key pressure waveform features were extracted and wave separation analysis was performed by combining aortic pressure and flow (Doppler echocardiography) data before and after the valve replacement.

Results: After the TAVI, aortic SBP and maximal blood flow velocity increased (from $134 \pm 22$ to $151 \pm 30 \mathrm{mmHg}, p=0.04$ and from $298 \pm 65$ to $376 \pm 81 \mathrm{~mL} / \mathrm{sec}, p<0.01$ respectively), while the ejection duration decreased (from $0.42 \pm 0.07$ to $0.35 \pm 0.06$ sec, $p<0.01$ ). A significant decrease in AIx and augmented pressure was noted (from $44 \pm 16$ to $17 \pm 12 \%$ and from $34 \pm 13$ to $17 \pm 14 \mathrm{mmHg}$ respectively, $p<0.01$ for all). Wave separation analysis however showed a comparable increase in both forward (from $61 \pm 14$ to $79 \pm 22 \mathrm{mmHg}, p<0.01$ ) and backward (from $31 \pm 11$ to $39 \pm 12 \mathrm{mmHg}, p<0.05$ ) wave amplitudes. Reflection magnitude and backward wave transit time remained unchanged ( $p=\mathrm{NS}$ ).

Conclusion: Decrease in AIx after TAVI is not associated with changes in wave reflection amplitude or timing. For patients with severe aortic stenosis, interpretation of AIx should be done with caution.

(C) 2019 Association for Research into Arterial Structure and Physiology. Publishing services by Atlantis Press International B.V. This is an open access article distributed under the CC BY-NC 4.0 license (http://creativecommons.org/licenses/by-nc/4.0/)
\end{abstract}

